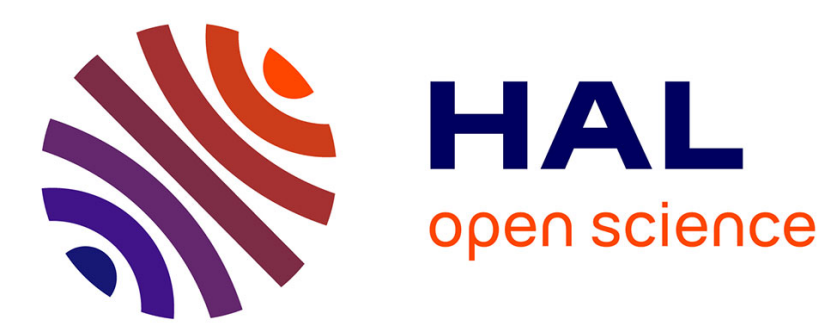

\title{
What is a reversed field pinch?
}

\author{
Dominique Escande
}

\section{- To cite this version:}

Dominique Escande. What is a reversed field pinch?. 2013. hal-00909102

\section{HAL Id: hal-00909102 \\ https://hal.science/hal-00909102}

Preprint submitted on 25 Nov 2013

HAL is a multi-disciplinary open access archive for the deposit and dissemination of scientific research documents, whether they are published or not. The documents may come from teaching and research institutions in France or abroad, or from public or private research centers.
L'archive ouverte pluridisciplinaire HAL, est destinée au dépôt et à la diffusion de documents scientifiques de niveau recherche, publiés ou non, émanant des établissements d'enseignement et de recherche français ou étrangers, des laboratoires publics ou privés. 


\title{
What is a reversed field pinch?
}

\author{
D. F. Escande \\ Aix-Marseille Université, CNRS, PIIM, UMR 7345, 13013 Marseille, France
}

\section{INTRODUCTION}

The reversed field pinch (RFP) is a magnetic configuration germane to the tokamak, that produces most of its magnetic field by the currents flowing inside the plasma; external coils provide only a small edge toroidal field whose sign is reversed with respect to the central one, whence the name of the configuration. Because of the presence of magnetic turbulence and chaos, the RFP had been considered for a long period as a terrible confinement configuration. Then strong enhancements were triggered in a transient way in the MST machine [1]. However, recently a change of paradigm occurred for this device. This was symbolically exhibited by the cover story of the August 2009 issue of Nature Physics: "Reversed-field pinch gets self-organized" [2]. Indeed, when the toroidal current is increased in the RFX-mod RFP in Padua (Italy), a self-organized helical state with an internal transport barrier (ITB) develops, and a broad zone of the plasma becomes hot (above $1 \mathrm{keV}$ for a central magnetic field above $0.8 \mathrm{~T}$ ).

The possibility of this helical state and of the corresponding improvement in confinement had been theoretically predicted. The present theoretical picture of the RFP mainly comes from three-dimensional nonlinear visco-resistive magnetohydrodynamic (MHD) simulations. They have exhibited dynamics having strong similarities with the experimental one, which triggered the experimental search for RFP states with improved confinement. The RFP ohmic state involves, as the nonlinear tearing mode, a helical electrostatic potential generating, as an electric drift, the so-called dynamo velocity field. The magnetic topology can bifurcate from a magnetic island to kink-like magnetic surfaces with higher resilience to magnetic chaos. This theoretical scenario was found to be relevant when ITB's enclosing a broad hot domain were discovered [3]. The ITBs occur in the vicinity of a maximum of the safety factor.

The new paradigm for the RFP supports its reappraisal as a low-external field, non-disruptive, ohmically heated approach to magnetic fusion, exploiting both self-organization and technological simplicity. The RFP comes with interesting transversal physics involving the other magnetic configurations. This chapter provides an introduction to the present knowledge about the RFP, and tries to bridge the large gap in its description that developed since the book by Ortolani and Schnack [4].

\section{SHORT DESCRIPTION}

As a device, the reversed field pinch (RFP) looks very much like a tokamak: a toroidally symmetric vacuum vessel is surrounded by a toroidally symmetric set of identical toroidal field coils; a central solenoid provides the loop voltage necessary to trigger a discharge, and to drive a current in the toroidal plasma inside the vessel. Furthermore a RFP may be operated as a tokamak, but a low field one. Indeed toroidal field coils provide only a small toroidal field (an order of magnitude smaller than the central one). The two magnetic configurations differ in several other respects:

- Most of the RFP magnetic field is produced by currents flowing inside the plasma, and for the same central toroidal field, the plasma current is an order of magnitude larger in the RFP than in the tokamak. As a result, the RFP equilibrium magnetic field has toroidal and poloidal components of comparable amplitudes. Their typical radial profiles are shown in Fig. 1.

- Since for the same central toroidal field, the plasma current is an order of magnitude larger in the RFP than in the tokamak, for the same resistivity in both configurations ohmic heating is two orders of magnitude larger in the RFP. While very high magnetic fields are required to reach thermonuclear temperatures in an ohmically heated tokamak (see sec. 5.2 of [5]), much smaller fields should be required for a RFP at parity of confinement. Therefore a RFP should be able to reach thermonuclear temperatures without any additional heating, a dramatic simplification. As yet no temperature saturation has been observed in the largest present RFP when current was increased up to 1.8 MA in optimized discharges to reach temperatures above $1.5 \mathrm{keV}$ (see Fig. 3 of [6]).

- The safety factor profile is typically decreasing. It starts from about $q_{0} \simeq a / 2 R$ in the center $(a$ and $R$ are the plasma minor and major radii) to reach a small value at the plasma edge related to the weak reversal of the toroidal field (see Fig. 2). Therefore in contrast to the tokamak, magnetic field lines in a RFP are almost poloidal. While the tokamak is close to a curved theta-pinch, the RFP is close to a curved Z-pinch.

-At variance with the tokamak, the toroidal current is pushed to values above the Kruskal-Shafranov threshold. This triggers a helical MHD instability (resistive kink) of the plasma annulus that nonlinearly saturates at finite amplitude with the help of a strong radial decrease of the toroidal field that reverses at the edge (see section XII C). 


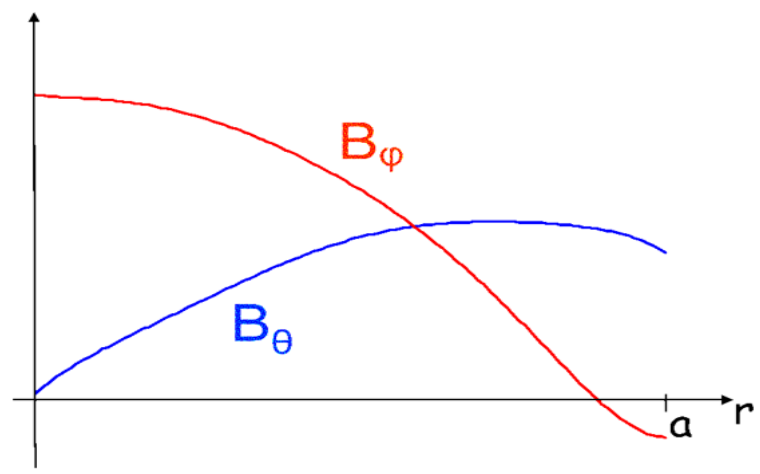

FIG. 1: Radial profile of the toroidal and poloidal magnetic fields in a RFP

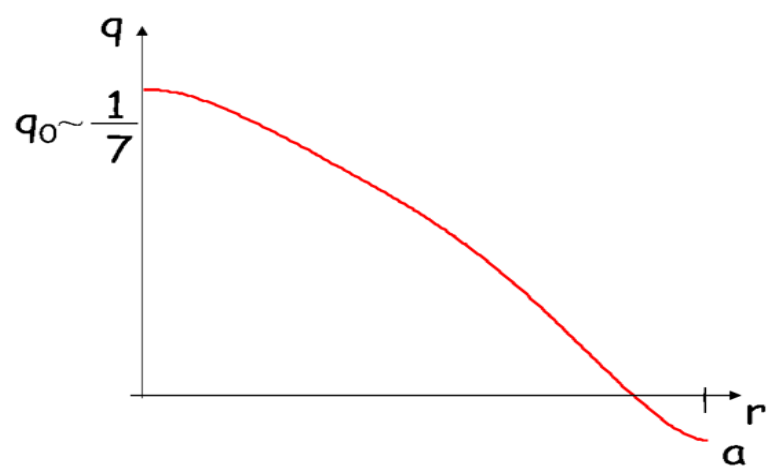

FIG. 2: Typical radial profile of the safety factor in RFX-mod

This self-organization mechanism makes the RFP configuration disruption-free because it corresponds to a full MHD relaxation of the magnetic field. This is at variance with the tokamak where the central toroidal field is maintained close to its edge value, but may relax disruptively.

- In the RFP the edge radial magnetic field needs to be controlled either by a thick shell or preferably by feedback controlled saddle coils [7]. This is important to avoid resistive wall modes which are unstable even at zero $\beta$, and to improve confinement. However this brings an additional complexity to the front-end with respect to a tokamak without magnetic ELM control, as explained in section V.

- The helical deformation due to the saturated kink makes RFP plasmas similar to stellarator ones. However the two configurations are lying symmetrically with respect to the tokamak, as far as magnetic self-organization is concerned: in a stellarator the magnetic field is almost completely defined by imposed outer currents, while in a RFP it is mostly produced by inner currents.

- Since the toroidal field coils only produce a weak outer negative toroidal field, the much stronger positive one in the plasma core is produced by poloidal currents inside the plasma. Since such currents cannot be driven by the toroidal loop voltage alone, a plasma flow is necessary for providing a $\mathbf{v} \times \mathbf{B}$ electromotive force in the poloidal direction. In other words a dynamo is acting in the plasma. As explained in section VIII, the plasma flow is now understood as a mere electrostatic drift due to the helical distortion of the plasma, a feature similar to that of a nonlinear tearing mode. From an astrophysicist viewpoint, the RFP dynamo is only half a dynamo since the toroidal current is directly driven by an externally imposed e. m. f..

There are two ways of producing strong magnetic fields with little heat dissipation: using currents in superconducting magnets, or in hot plasmas: the resistivity of a $20 \mathrm{keV}$ plasma is about one tenth of that of copper at room temperature. The RFP has the unique feature among confinement devices to choose this second path. The remnant ohmic dissipation turns out to be useful to reach thermonuclear temperatures. This is in contrast with the power used to operate the cryogenic system required by superconducting magnets. In the latter case one must extract ohmic and nuclear heat out of the "refrigerator"!

Finally since the outer magnetic field applied to a RFP plasma is weak, this configuration comes close to a minimum 
requirement for the virial theorem, which states that self-confinement of a magnetized plasma by inner currents is impossible, and that some currents flowing in outer conductors are necessary. This is in contrast with the tokamak and the stellarator where the outer and inner magnetic fields have a comparable amplitude.

Presently the largest RFP is the RFX-mod device $(\mathrm{R}=2 \mathrm{~m}, \mathrm{a}=0.459 \mathrm{~m}$, maximum current $2 \mathrm{MA})$ in Padua (Italy); this corresponds to a $10 \mathrm{~m}^{3}$ plasma with a maximum central magnetic field above $1 \mathrm{~T}$ [8]. Other present machines are MST $(\mathrm{R}=1.5 \mathrm{~m}, \mathrm{a}=0.5 \mathrm{~m}$, nominal current 0.6 MA) in Madison (USA), EXTRAP T2R ( R = 1.24 $\mathrm{m}, \mathrm{a}=0.18 \mathrm{~m}$, nominal current $300 \mathrm{kA}$ ) in Stockholm (Sweden), and RELAX $(\mathrm{R}=0.51 \mathrm{~m}, \mathrm{a}=0.25 \mathrm{~m}, \mathrm{nominal}$ current $80 \mathrm{kA}$ ) in Kyoto (Japan). The KTX machine is under construction in Hefei (China) with a size close to MST and RFX and should operate in 2015.

\section{USEFULNESS OF THE RFP CONFIGURATION FOR FUSION SCIENCE AND DYNAMO PHYSICS}

The progress in tokamak performance was an incentive to design and to build ITER, in order to address the issue of burning thermonuclear plasmas. This project should be followed by a demonstration fusion plant called DEMO. However, important physics issues need to be solved both for ITER and for the definition of DEMO: what is the origin of the Greenwald density limit [9], how do transport barriers form and stay, what is the origin of plasma rotation, what is the effect of additional heating, how to scale reactor parameters out of smaller experiments, how dangerous may be fast particle driven MHD modes, what is the role of ambipolar electric fields, what is the benefit of radiating layers, of a helical deformation of the magnetic field, etc..? RFP experiments may bring transversal views about all these issues and help sorting out their many interpretations in the tokamak frame. They may bring a similar help to stellarator physics too.

In particular, understanding the density limit in magnetic confinement might enable to come closer to this limit or to overcome it, would increase considerably the reactivity of thermonuclear plasmas, which would dramatically increase the prospects of magnetic fusion. Important progress in this direction might be done by taking advantage that this limit is the same Greenwald limit in the tokamak and the RFP. With respect to the tokamak and the stellarator, the RFP has a low imposed external field. It has a helical magnetic field like the stellarator, but it is more self-organized than a tokamak, and much more than a stellarator.

Apart from physics issues, there are also operational issues in common with the tokamak like the control of MHD modes [7]. Some coordinated activity already brought fruitful results like the possibility of controlling the $(2,1)$ mode in tokamaks and of lowering $\mathrm{q}(\mathrm{a})$ below $3[10]$. Another possibility yet to explore would be to turn a disruptive discharge in a tokamak into a RFP one.

Since (half) a dynamo is acting in the RFP, there is a natural resonance with the astrophysical dynamos. The corresponding communities have been interacting for several years, in particular in the frame of the Center for Magnetic Self-Organization (CMSO) in the United States. The von Karman Sodium (VKS) experiment in Cadarache came with a striking result: an incompressible fluid dynamo can drive a RFP magnetic state all by itself! This experiment studies dynamo action in the flow generated inside a cylinder filled with liquid sodium by the rotation of coaxial impellers (von Karman geometry). It evidenced the self-generation of a stationary dynamo when the impellers do not rotate with the same angular velocity, when at least one is made of a ferromagnetic material, and when the velocity fluctuations are rather small. Furthermore the dynamo with two rotating impellers is not the superposition of that with single rotating impellers. The magnetic field averaged over a long enough time corresponds to a RFP magnetic state with a large $m=0$ mode (see Fig. 7 of [11]). It is striking that the incompressible turbulent flow produced by the impellers leads to the same magnetic equilibrium as in a current driven pinch whose plasma is compressible. Understanding the universality of the configuration might lead to a large leap forward of dynamo theory.

\section{ATTRACTIVITY OF THE RFP CONFIGURATION FOR A REACTOR}

We just explained that the RFP configuration is disruption-free and should be able to reach thermonuclear temperatures without any additional heating. Here are further attractive aspects of a possible RFP reactor:

- Since most of the magnetic field is produced by currents flowing inside the plasma, the maximum magnetic field is not bounded by the maximum value imposed by superconductor technology like for tokamaks and stellarator, but by the ability to drive a high plasma current. A priori this should enable to reach higher magnetic fields than provided by Nb-Sn cables. Therefore improving performances may be sought not only by increasing the size of the device, but also by increasing its current.

- Since most of the magnetic field is produced by currents flowing inside the plasma, copper toroidal field coils might be used. This also lowers the requirements on the thickness of their neutron shielding, and on the growth of 
the radial build up. The coils are also subjected to lower forces than in a tokamak or a stellarator, which decreases the mechanical constraints. All this would bring a dramatic technological simplification coming with large money savings. However a superconducting central solenoid might still be advisable.

- $\beta$ 's as high as 0.25 are already possible, which reduces the amplitude of the confining magnetic field for a given thermonuclear reactivity. With the low external toroidal field this means a high engineering beta (it is already up to $26 \%$ in present experiments).

- The RFP configuration is relatively compact, which leads to a high mass power density, and possibly to a singlepiece maintenance thanks to the low field toroidal field coils.

- This comes with efficient assembly and disassembly, and possibly a free choice of aspect ratio.

- Since the RFP does not suffer from disruptions, fast ramp-up and ramp-down of the current are possible. This opens the prospect of a non-stationary reactor where rapidly succeeding (on the order of $1 \mathrm{~s}$ ) current flat-tops with opposite signs of the current would be produced by a double swing poloidal circuit. Indeed the rapid succession of flat-tops would minimize the mechanical fatigue of materials due to thermal cycles which is a concern for tokamaks, since ramp down and ramp up must be slow to avoid disruptions.

- Though non axis-symmetric, the magnetic field should not require optimization like in the stellarator. Indeed, because of ohmic heating, alpha particles do not need to transfer their energy efficiently to the plasma center. Furthermore the loss of axis-symmetry decreases when going towards the plasma edge, which decreases the trapped fraction of fast particles and the related transport. Finally with the amplitude of the helical field seen in experiments, super-banana orbits are present in negligible fraction, which helps fast particles to be better confined [12]. All this should prevent alpha particles to be lost rapidly to the wall.

- Because of ohmic heating, the enhancement of alpha particle transport due to unstable Alfvenic or geodesic acoustic modes is less an issue than in the tokamak or the stellarator.

These features would lead to a simpler, more robust, and less expensive reactor than the tokamak or stellarator configurations. It, is worth recording that, for ITER, disruptions are the main physical challenge, the neutral beam heating system is still under development, and the large superconducting coils are a very expensive high tech part of the machine whose failure would require a long shut down, especially whenever the machine would be nuclearly activated.

\section{CHALLENGES AHEAD}

As just explained, the RFP configuration has many potentially interesting features for a thermonuclear reactor. However it must face several challenges to become a credible candidate. Fortunately there is no first-principles-based showstopper. The main challenge for the RFP is the quality of confinement, since its helical magnetic self-organization comes with a magnetic chaos which is as yet higher than in a stellarator. Improvements are now sought by a better control of the edge magnetic field [13, 14], and of plasma-wall interaction [15], and by a further increase of the plasma current.

Another challenge is core fueling, since wall-recycling is not efficient enough to provide high densities at high plasma current [15]. Pellet injection needs a fine tuning, since big pellets decrease too much the bulk temperature, while small ones do not feed the plasma center. The need for this fine tuning is a consequence of the self-organized feature of the RFP plasma.

A path to escape the constraints of full self-organization of the RFP plasma is to drive the plasma either impulsively or by sustained current drive [17, 18]. This line of research is being very active on the MST machine [19].

The need of feedback controlled saddle coils to control the edge radial magnetic field brings an additional complexity to the front-end with respect to a tokamak. RFX-mod has 192 saddle coils to avoid resistive wall modes and to provide adequate boundary conditions to the helical magnetic field inside the plasma. The number of such coils that would be necessary for a RFP reactor is still an open issue, but is likely to be higher than the one considered for ELM control in ITER. The peak electric power used by the RFX feedback control system is on the order of $25 \%$ of the ohmic power, but the mean power is about $10 \%$.

\section{LAWSON CRITERION}

Reaching thermonuclear temperatures without any additional heating means that a RFP cannot work in the ignition regime. Indeed decreasing ohmic heating would mean decreasing the magnetic field and confinement too. This makes the usual "Lawson criterion" of tokamaks and stellarators irrelevant for the RFP.

In order to yield a net production of electricity a RFP power station should verify condition $e\left(P_{\mathrm{ohm}}+P_{\text {fusion }}(1+\right.$ $\left.\left.m_{\text {blanket }}\right)\right)>P_{\text {ohm }}+P_{\text {coils }}$ where $P_{\text {ohm }}, P_{\text {fusion }}$ and $P_{\text {coils }}$ are respectively the ohmic power, the power produced by 


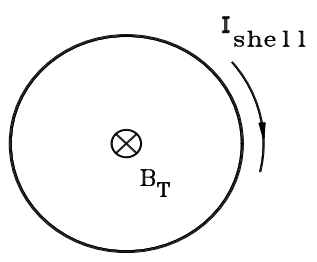

(a)

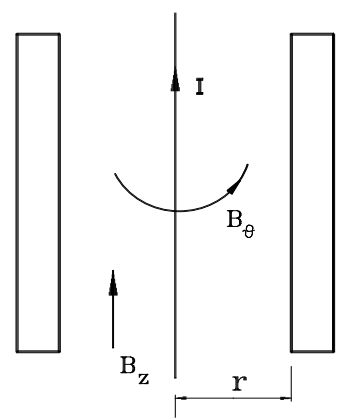

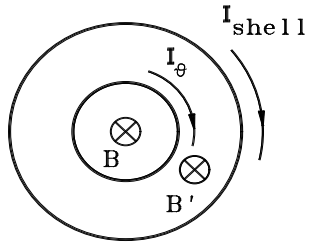

(b)
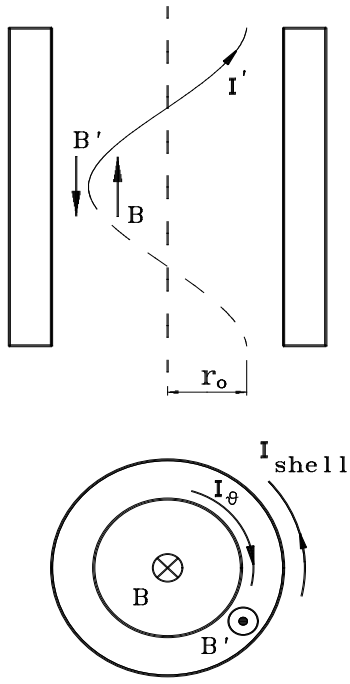

(c)

FIG. 3: Wire model (reproduced by permission from [22] doi:10.1088/0741-3335/42/12B/319)

fusion reactions, and the power dissipated in the coils and other electrical devices of the reactor; $e$ is the power station efficiency and $m_{\text {blanket }}$ the multiplication factor of the fusion power due to exothermic nuclear reactions in the tritium breeding blankets. This implies

$$
P_{\text {fusion }}>\left[(1 / e-1) P_{\mathrm{ohm}}+P_{\text {coils }} / e\right] /\left(1+m_{\text {blanket }}\right)
$$

The total energy in the plasma is $W=3\langle n T\rangle V$ where $n$ is density and $T$ the temperature, $V$ the plasma volume, and the brackets mean "volume averaging over $V$ ". Let $\tau_{\mathrm{E}}$ be the energy confinement time. Then the power loss $W / \tau_{\mathrm{E}}$ should be balanced by ohmic heating and alpha heating in a stationary regime: $W / \tau_{\mathrm{E}}=P_{\mathrm{ohm}}+P_{\text {fusion }} / 4$. Using eq. (1) this yields $P_{\text {fusion }}\left(3 / 4+m_{\text {blanket }}+1 / 4 e\right)>(1 / e-1) W / \tau_{\mathrm{E}}+P_{\text {coils }} / e$. Taking into account the fact that $P_{\text {fusion }}$ scales like $n^{2}$, this condition yields again a condition on $n \tau_{\mathrm{E}}$ which is reminiscent of that originally derived by Lawson which did not consider ignition [20]. The minimum value of $n \tau_{\mathrm{E}}$ depends on the choice of the parameters. Neglecting $P_{\text {coils }} / e$, for $m_{\text {blanket }}=0.2$, and $e=1 / 3$, one finds a value about 1.2 times the classical value for ignition in a tokamak $1.510^{20} \mathrm{~m}^{-3} \mathrm{~s}$ (Sec. 1.5 of [5]). This value decreases if the efficiency increases.

\section{INTUITIVE MODEL OF MAGNETIC SELF-REVERSAL}

Since magnetic field reversal may sound mysterious it is useful to first introduce a simple a toy model which makes it intuitive [21, 22]. Consider a current-carrying resistive wire initially placed on the axis of a cylindrical flux conserver (see Fig. 3). A finite, but small, axial magnetic field is present inside the cylinder. This means an azimuthal current is flowing in the cylinder (Fig. 3 (a)). The wire is in unstable equilibrium, and a small perturbation triggers a kink. Imagine there is a "demon" forcing the absolute value of the pitch, but not its sign. Then the kink develops with a pitch whose sign is such that the azimuthal part of the current flowing in the wire has the same orientation as the one in the cylinder, which brings a mutual attractive force (Fig. 3 (b)). Such a pitch brings also a solenoidal effect which increases the magnetic field and flux inside the kinked wire. The flux conserver imposes accordingly a decrease of the magnetic field and flux outside. As long as the current in the cylinder keeps its sign, the instability cannot quench. 


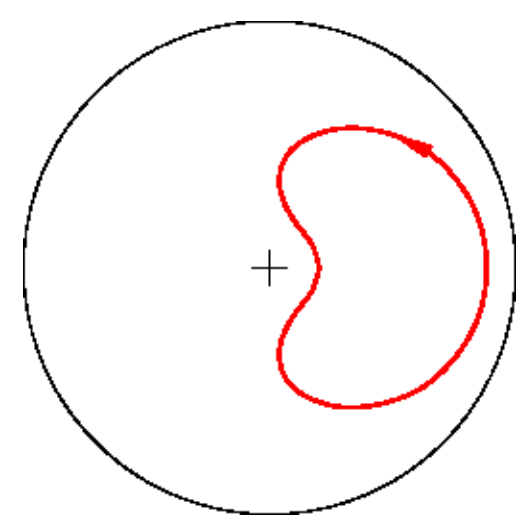

FIG. 4: Helical magnetic surface

The continuing growth of the magnetic field and flux inside the kinked wire forces the outer magnetic field and the current in the cylinder to reverse. Eventually the wire finds an equilibrium where it is trapped in a sheared magnetic field (Fig. 3 (c)). This model exhibits a self-organized magnetic system with field reversal where the loss of cylindrical symmetry is essential. It is a variant of a model for the saturation of the ideal kink proposed by Kadomtsev where the wire is superconducting, and where the current vanishes at saturation [23].

One of the scenarios for a RFP discharge, "self-reversal scenario", is quite similar to that of the above wire model. We show later that this picture is confirmed by two dimensional resistive MHD numerical simulations of the RFP, which are usually run with the axial flux conservation rule. Since the RFP is a self-organized magnetic system resulting from the nonlinear saturation of a (resistive) kink mode involving field reversal, it turns out to be resilient to disruptions. In RFP experiments, aided reversal is generally preferred to self-reversal in order to save volt-seconds and to help the formation of the poloidal part of the current [24]. However most of the current corresponds to the paramagnetic pinch component of the configuration as discussed in Sec. XII C.

\section{INTUITIVE DESCRIPTION OF THE DYNAMO}

Another element of RFP physics which may sound mysterious is the dynamo process underlying the configuration. This process turns out to be a mere consequence of magnetic relaxation, as is revealed by nonlinear visco-resistive MHD simulations of the RFP where stationary pure single helicity (SH) states are found [25]: (i) Since the toroidal and poloidal magnetic fields have the same order of magnitude in a RFP, the current flowing in the plasma is much higher than in a tokamak for the same field; indeed $q \ll 1$ over the whole cross-section of the plasma. This means that the Kruskal-Shafranov threshold is crossed during the start-up phase of the discharge. In analogy with the wire model, a resistive kink develops, bringing a helical distortion of the magnetic field (see Fig. 4) (ii) This distortion requires a modulation of the current density along current lines. (iii) This modulation requires the existence a modulated electric field, which must be electrostatic since the SH state is stationary. (iv) The corresponding electrostatic potential brings a component of the electric field perpendicular to the magnetic field. ( $\mathrm{v}$ ) This component drives an $\mathbf{E} \times \mathbf{B}$ motion whose non axis-symmetric part is exactly the dynamo velocity field. This picture is backed up by the analytical calculations of Secs. XIIB and XIIE.

In reality such a description holds for any plasma column with a single helicity perturbation of the magnetic field. This holds in particular for a saturated tearing mode. Therefore the RFP dynamo belongs in the same family as the tearing mode dynamo.

\section{NECESSITY OF A HELICAL DEFORMATION}

Up to this point the RFP configuration was linked with a helical deformation of the plasma. We now show that there cannot be an axis-symmetric RFP.

Imagine there is an axis-symmetric RFP with a vanishing pressure gradient next to the reversal radius $r_{\mathrm{r}}$. Then the pitch of current lines reverses together with that of the magnetic field at $r_{\mathrm{r}}$. For a given loop voltage the poloidal current must reverse at the reversal radius, since the sign of the toroidal current is fixed. But this implies the toroidal field is minimum at $r_{\mathrm{r}}$ (see Fig. 5), which contradicts our assumption that it reverses. A v $\times \mathbf{B}$ e. m. f. cannot help 


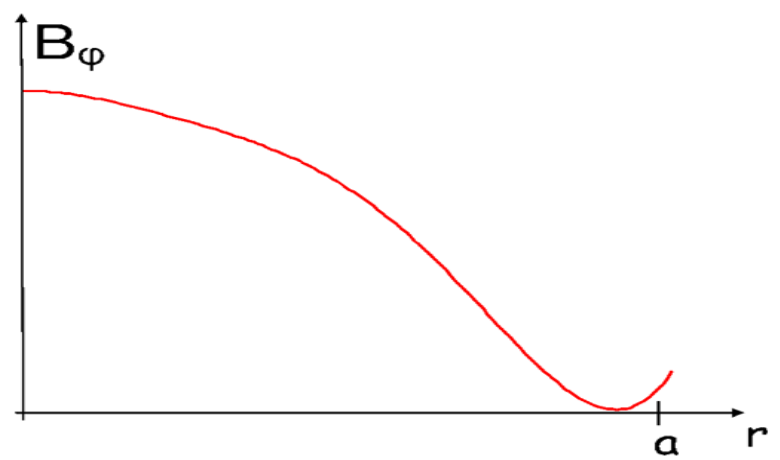

FIG. 5: Impossibility of reversal in a cylindrical RFP

since $\mathbf{B}$ is poloidal at reversal. If there is a finite pressure gradient next to $r_{\mathrm{r}}$, this modifies only the toroidal current at reversal, and leaves unaffected the reasoning on the poloidal current.

Because of the importance of this result, we prove it again by an alternative method. If an axis-symmetric RFP would exist, a finite azimuthal current would be necessary at $r_{\mathrm{r}}$ to produce the reversal of the toroidal field. However it could not be driven neither by the inductive electric field due to the loop voltage, nor by a $\mathbf{v} \times \mathbf{B}$ e. $\mathrm{m}$. f. since $\mathbf{B}$ is poloidal at reversal.

Therefore no dynamo can sustain an axis-symmetric RFP. This statement is germane to Cowling's theorem in astrophysics [26] which states that no axis-symmetric dynamo can maintain a symmetric magnetic field. By extension one often speaks of a "Cowling theorem" for the RFP. The wire model shows that the RFP fulfills this theorem in a quite natural way: field reversal is a consequence of the plasma kink, and thus of the loss of axis-symmetry. From both its magnetic and velocity fields, the RFP may be viewed as a helically distorted paramagnetic pinch. As shown in [27], the helical deformation of the plasma is already present for ultra-low safety factor configurations.

\section{MHD SIMULATIONS}

\section{A. From single to multiple helicity}

The analytical description of the RFP configuration is still at its infancy, but a lot of information about RFP self-organization has been provided by numerical simulations using the visco-resistive compressible nonlinear MHD model in the constant-pressure, constant-density approximation [4, 28-30]. Though its limited physics contents, this model displays a dynamics with strong similarities with the experimental one.

The first theoretical proof of existence of SH states was their discovery in 1990 in 3D numerical simulations where no helicity was a priori forced upon the dynamics [28, 31-33]. Later on, the existence of SH states was understood as corresponding to the high dissipation limit of the system [29,34], as defined by the product $d$ of the central viscosity and resistivity (the corresponding dimensionless number is the Hartmann number scaling as $1 / \sqrt{d}$ ). When $d$ decreases, secondary modes with other helicities develop in the system. Together with this, the magnetic configuration becomes time-dependent, and switches intermittently between two fluctuating states: a quasi SH (QSH) state where secondary modes are smaller than the original one, and a multiple helicity $(\mathrm{MH})$ state where at least two modes have similar amplitudes. When $d$ decreases, the duration of the QSH phases decreases, as well as the percentage of time where QSH dominates. As will be shown in section XI the numerical scenario is quite similar to the experimental one in RFX-mod: a decrease of $d$ in the former is equivalent to a decrease of the current in the latter, and switches the transition from QSH to MH states. Historically the numerical scenario was an incentive to revisit the RFX data base in 1999. Shots with long lasting QSH states were found to be present for quite a time in the data base [35], but they had been neglected because they were out of the traditional MH paradigm of the machine!

When performed in toroidal geometry, 3D nonlinear visco-resistive MHD simulations show that toroidal coupling prevents the system to reach a pure SH state when $d$ increases, but that magnetic chaos due to toroidal coupling stays limited in SH states for the aspect ratios of the largest present RFP's [36].

On top of the bifurcation leading from $\mathrm{MH}$ to $\mathrm{SH}$, there is another one which modifies the magnetic topology. When the amplitude of the $\mathrm{SH}$ mode is low enough, there is a magnetic island. Therefore the magnetic field displays two magnetic axes: the unperturbed axis-symmetric one and the one related to the island O-point. When the amplitude 
of the SH mode increases there is a bifurcation that changes the topology of the magnetic field through the coalescence of the axisymmetric O-point with the island X-point [35]. Then the former island O-point becomes the only magnetic axis, and the magnetic topology is like that of a non resonant kink. This coalescence may be intuitively understood by noting that an increase of the SH mode means that the inner loop of the separatrix becomes tighter and tighter about the former axisymmetric O-point and vanishes when its inner area vanishes. Then the safety factor $q$ profile goes through a maximum located in the vicinity of the former separatrix [37]. Furthermore the resilience to chaotic perturbations of a one parameter one degree of freedom Hamiltonian dynamics increases when its corresponding separatrix vanishes due to a saddle-node bifurcation. As a result, SH states without separatrix are more resilient to chaos than those with a magnetic island [35, 38-40].

The next subsections provide a more precise picture of the results provided by the visco-resistive compressible nonlinear MHD model in the constant-pressure, constant-density approximation [28-30]. Its equations are

$$
\begin{gathered}
\frac{\partial \mathbf{B}}{\partial t}=\nabla \times(\mathbf{v} \times \mathbf{B})-\nabla \times(\eta \mathbf{J}), \\
\frac{\partial \mathbf{v}}{\partial t}+(\mathbf{v} \cdot \nabla) \mathbf{v}=\mathbf{J} \times \mathbf{B}+\nabla^{2}(\nu \mathbf{v}),
\end{gathered}
$$

where $\eta$, and $\nu$ are the radial distributions of respectively the resistivity and of the kinematic viscosity, and where $\mathbf{J}=\nabla \times \mathbf{B}$ and $\nabla \cdot \mathbf{B}=0$. Here $\mathbf{B}$ is normalized to the value $B_{0}$ of the axial magnetic field on axis, time and velocity are normalized to the Alfvén time $\tau_{A}$ and velocity $v_{A}$ respectively computed with $B_{0}$, and the position to the plasma radius $a$. In these units $\eta$ is the inverse Lundquist number, $\eta=\tau_{A} / \tau_{R} \equiv S^{-1}$, and $\nu=\tau_{A} / \tau_{V} \equiv R_{m}^{-1}$. The RFP is simulated as a straight periodic cylinder with axial periodicity $2 \pi R$.

The plasma current and the axial magnetic flux are taken as constant, which implies the constancy of the pinch parameter $\Theta \equiv B_{\theta}(a) /\left\langle B_{z}\right\rangle$ (in this paragraph the averages are done over the toroidal and poloidal angles). This type of model has been widely used for RFP simulations (see [4] and [34], and references therein). Usually numerical simulations start from a paramagnetic pinch state with $B_{z}(a)$ close to 0 . For $\Theta \gtrsim 1.55$ the system which is (resistive) kink unstable, relaxes toward a RFP state where the reversal parameter $F \equiv B_{z}(a) /\left\langle B_{z}\right\rangle$ is in the range $0<F<-0.5$.

\section{B. Single helicity}

The possibility of having a RFP plasma in a pure SH state was put forward since 1983 through two-dimensional numerical simulations [41-45] with $\beta=0$ where a stationary RFP state was found by retaining only one ratio $m / n$ for the Fourier harmonics beyond $(0,0)$, and thus forcing the final RFP state to be in the SH state. Some indication of this possibility was already present in the first numerical simulation of the RFP [46]. In the simulations, the magnetic field reversal is much smaller than in the wire model, as only a small part of the axial current is involved in the drive of the helical deformation.

Let $\eta_{0}=\eta(0), \nu_{0}=\nu(0), \bar{\eta}=\eta / \eta_{0}$, and $\bar{\nu}=\nu / \nu_{0}$. Applying to equations (2) and (3) the rescaling $t \rightarrow \bar{t}=\sqrt{\frac{\eta_{0}}{\nu_{0}}} t$ with the corresponding rescaling of velocity $\mathbf{v} \rightarrow \overline{\mathbf{v}}=\sqrt{\frac{\nu_{0}}{\eta_{0}}} \mathbf{v}$ yields

$$
\begin{gathered}
\frac{\partial \mathbf{B}}{\partial \bar{t}}=\nabla \times(\overline{\mathbf{v}} \times \mathbf{B})-\nabla \times\left(H^{-1} \bar{\eta} \mathbf{J}\right), \\
P^{-1}\left[\frac{\partial \overline{\mathbf{v}}}{\partial \bar{t}}+(\overline{\mathbf{v}} \cdot \nabla) \overline{\mathbf{v}}\right]=\mathbf{J} \times \mathbf{B}+\nabla^{\mathbf{2}}\left(H^{-1} \bar{\nu} \overline{\mathbf{v}}\right),
\end{gathered}
$$

where $P=\nu_{0} / \eta_{0}$ is the magnetic Prandtl number, and $H=\left(\eta_{0} \nu_{0}\right)^{-1 / 2}$ is the Hartmann number. The rescaled dynamics depends only on the value of the Hartmann number when the inertia term becomes negligible. This happens to be the case for a large range of simulation parameters. Indeed simulations reveal that $H$ is the right control parameter independently of $P$ and $\Theta[29,34]$ (see also figure 6); there the aspect ratio was $R / a=4$. The Hartmann number was introduced in the fusion context in $[47,48]$, and is present implicitly through the product $d$ of dissipations introduced in Sec. X A in [49] and in [50] for a bifurcation approach of tearing modes.

Therefore the previous "high viscosity" simulations turn out to be in fact "high dissipation" or "small $H$ " simulations. The upper bound in $H$ to reach $\mathrm{SH}$ is about 1000. As usual in nonlinear dynamics an increase of dissipation (decrease of $H$ ) is favorable to a laminar behavior of the system. Depending on the initial conditions, two nearby different helicities were found to be selected by the plasma when relaxing to SH [29, 34]. The corresponding magnetic states are stable fixed points of the dynamics defined by Eqs. 2 and 3 . 

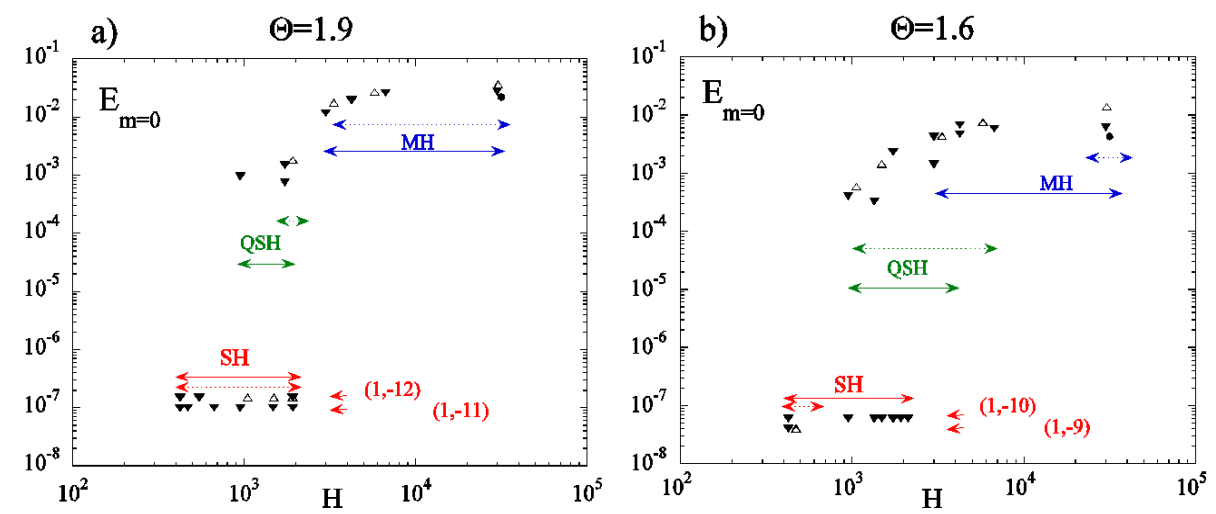

FIG. 6: Transition diagrams at two values of the pinch parameter: a) $\Theta=1.9$, b) $\Theta=1.6$. $E_{m=0}$ is the time averaged magnetic energy of the $m=0$ modes and $\mathrm{H}$ is the Hartmann number. The symbol $\triangle$ corresponds to $S=3.310^{3}$ with $P=[0.012,50]$, $\boldsymbol{\nabla}$ to $S=3.010^{4}$ with $P=[1,5000]$, and $\bullet$ to $S=10^{5}$ with $P=10$. The vanishing $\mathrm{SH} m=0$ modes energy is represented as a finite conventional value with different offsets associated to the different preferred helicities (reproduced by permission from [34] doi:10.1088/0741-3335/46/12B/027).

These numerical simulations enabled the description of the dynamo provided in section VIII $[25,51,52]$. The electric field $\mathbf{E}$ in the SH state can be computed from the simulation variables by using Ohm's law 14. It is curl-free, since the final state is stationary. Its divergence provides an electric charge distribution which takes on a dipolar helical structure, and whose amplitude is small, in agreement with the assumptions leading to MHD equations. Then the perpendicular part of the velocity is provided by

$$
v_{\perp e s}=-\nabla \phi \times \frac{\mathbf{B}}{\mathrm{B}^{2}} .
$$

Since $\nabla \times \mathbf{E}=0$, then $\partial E_{z} / \partial r=\partial E_{r} / \partial z$ and the $2 \pi R$ periodicity in $z$ imply that the average over $z$ of $E_{z}$ is a constant $E_{0}$ over space.

Numerical simulations bring the new information that the velocity field in the SH state is topologically equivalent to the radial pinch velocity field of the paramagnetic pinch, as given by Eq. (41) for an axis-symmetric magnetic field. The SH field corresponds to a helically distorted version of the paramagnetic one, where the pinch is now toward the helical magnetic axis. Therefore the dynamo corresponds to a velocity field which is rather confining. In MHD codes where density is free to evolve, such a pinch velocity leads to a build up of the plasma density on the helical axis [53]. In a genuine plasma this triggers some transport mechanism opposing the growing density gradient. This effect is modified in turn by a pressure gradient, and by heat transport.

\section{Multiple helicity}

When $H$ increases (dissipation decreases), another helicity becomes unstable in the previous SH equilibrium, and after a transition region for $10^{3} \lesssim H \lesssim 10^{4}$ to be described later, the system enters a non stationary MH regime $[29,31-33]$. This is shown in figure 6 where the energy of the $m=0, n \neq 0$ modes is used as an order parameter for the SH-MH transition; indeed these modes vanish in the SH state, since they result of the beating of at least two different helicities. From the point of view of nonlinear dynamics this regime corresponds to a new basin of self-organization. A lot of numerical simulations have been devoted to the $\mathrm{MH}$ regime (see $[4,28]$ for a review). Most of the features reported below about MH can be found in [28]. Spectral analysis of the MH state for $H>10^{4}$ reveals that several $m=1$ modes with different axial mode numbers $n>0$ and comparable amplitudes are simultaneously present in the plasma, and that the $m=1$ mode spectrum extends up to the maximum available $n$ number of the simulation, but with a decreasing amplitude. This yields a broad range of values to the safety factor $m / n$ of the modes. As in SH, the harmonics of the $m=1$ modes are present too. Since equations (2-3) are nonlinear, the beating of these modes produces a wide spectrum of modes with other $m$ 's, and in particular $m=0, n \neq 0$ modes. All Fourier modes display temporal fluctuations. Indeed it is impossible to solve the parallel Ohm's law (Eq. 16) for $\phi$ if MH is assumed for the spatial modulation of a static magnetic field [33]: a static $\mathrm{MH}$ ohmic state is impossible.

A large helical deformation of magnetic field lines is present in a finite axial (toroidal) domain. As a result the spatial modulation of the magnetic field is far from being random, even if the temporal fluctuations of each mode 
look turbulent: the nonlinear interaction drives a strong mode locking among the $m=1$ modes. Order is also found temporally when monitoring the reversal parameter $F$. Indeed $F(t)$ displays a series of oscillations whose pseudoperiod is about $\tau_{R} / 10$. The phases where $F(t)$ decreases (increase of magnetic field reversal) are more rapid than those where it increases $[28,54]$. The amplitude of the oscillations grows with $\Theta$ as in spheromak simulations [36]. They are called fast relaxation events. The fast relaxation events come together with a higher amplitude of the magnetic fluctuations, which shows that a solenoidal effect of the helical deformation is present in MH too. They correspond to the formation of thin current sheets with a rapid energy dissipation. The amplitude $\delta B$ of magnetic fluctuations is found to scale like [55]

$$
\delta B \sim H^{-\frac{1}{4}}\left(1+P^{-1}\right)^{\frac{1}{8}},
$$

consistently with the Sweet Parker expectation [28, 56]. For MH regimes confinement scaling is rather poor [57].

An important consequence of the $\mathrm{MH}$ character of the magnetic field spectrum is the fact that the dynamics of field lines is no longer integrable, and that magnetic chaos sets in [58, 59]. The magnetic mode spectrum corresponds mainly to $q \geq 0$ modes. Therefore the corresponding magnetic chaos is present in this domain when the field lines are reconstructed from the code outputs. This domain does not correspond to a well-defined domain in $r$ because of the above-mentioned large helical deformation which brings a bulging of chaos at its axial position. In the $q>0$ domain no magnetic island is visible. However for $q=0$ a chain of magnetic islands is present. The $q>0$ magnetic chaos bulges through one of the X-points of this island chain. The $m=0$ mode increases chaotic transport in the $q \gtrsim 0$ domain, but the $m=0$ chain of islands decreases the size of the chaotic domain, and plays the role of a transport barrier in the chaotic region [34,60]. Equation (7) shows that the amplitude of magnetic fluctuations increases when $H$ decreases. This does not mean that chaos is steadily increasing. Indeed when $H$ decreases, at some moment the system reaches the SH state where the single helicity nature of the magnetic field makes the magnetic field lines non chaotic.

The large scale magnetic chaos of MH states has an interesting consequence, as was shown by Rusbridge [61] on the basis of an idea by Kadomtsev and Moffatt: (i) In the radial domain where the magnetic field is chaotic, transport is fast, and the equilibrium is almost force-free; therefore $\mathbf{J}=\mu \mathbf{B}$ where $\mu$ may be space-dependent. (ii) Setting this in $\nabla . \mathbf{J}=0$, implies $\nabla \mu . \mathbf{B}=0$, which shows that $\mu$ must be constant along field lines; thus $\mu$ is constant in the chaotic radial domain. This straightforward derivation yields a result in full agreement with the fact that in MHD simulations $\mu$ is almost constant in most of the $q>0$ domain, but not outside [62]. In the past this domain of almost constant value of $\mu$ was rather considered as a hint of the validity of Taylor relaxation theory [63].

\section{Quasi single helicity}

When $H$ increases, the transition from $S H$ to $M H$ turns out to be continuous, in analogy with a second order phase transition [29]. In the vicinity of $H=2000$ the system displays a temporal intermittency whose laminar phases are of quasi SH (QSH) type. Then the $m=1$ magnetic field fluctuations spatial spectrum is made up almost entirely by one individual MHD mode. SH or MH transients may last over long times [64]. The temporal intermittency in the transition from $\mathrm{SH}$ to $\mathrm{MH}$ is like critical opalescence in a second order phase transition, and is the signature of a bifurcation of the dynamical system of interest.

As a result of the continuity between $\mathrm{SH}$ and $\mathrm{MH}$ states, the large axially localized helical deformation in the latter is the result of a continuous deformation of the axially uniform helical shape of the former. The continuity bears on the dynamo as well. Indeed, though a fluctuating inductive electric field be present in the MH state, the velocity field is still dominated by the electrostatic contribution $[25,51,52]$. Magnetic chaos sets in progressively during the transition too.

\section{EXPERIMENTAL RESULTS}

Historically, the RFP configuration was discovered in an empirical way [65]. During three decades the plasmas were found in a state of magnetic turbulence. In such a state weak confinement resulted from the chaos of magnetic field lines and strong plasma-wall interaction due to a toroidally localized large helical deformation of the plasma. Since 1993 plasmas with transient states where magnetic chaos decreases were found in the data base of all large RFP's. In 1999 these states were found to last most of the flat top of discharges with a current about 1 MA in the RFX machine, but in a non stationary way. After an upgrade of RFX which provided in particular a good control of the edge radial magnetic field, higher currents were reached and a topological bifurcation occurred in the magnetic field providing a broad hot central domain bounded by an internal transport barrier [2, 68]. The following subsections address these successive steps. 


\section{A. Multiple helicity}

At low current, measurements display a wide spectrum of magnetic fluctuations. The various spatial Fourier modes are temporally fluctuating too. They correspond to resistive kink and tearing modes. Many $m=1$ and $m=0$ modes with different toroidal mode numbers $n$ and comparable amplitudes are simultaneously present in the plasma, which motivates to qualify this regime Multiple Helicity (MH). A locking is observed between these modes, leading to a toroidally localized large helical deformation (LHD) of the plasma and of the magnetic field lines. As a result the plasma bulges and has a localized strong interaction with the first wall.

Images of the plasma core obtained through soft X-ray (SXR) tomography display a poloidally symmetric emissivity. This indicates that a wide region of strong heat transport is present in the plasma core, in agreement with the existence of a chaotic magnetic field. The temperature gradient is localized in the domain where the toroidal field reverses.

\section{B. Quasi single helicity}

Transient states where the magnetic field fluctuations spatial spectrum is dominated by one individual $(m=1, n \sim$ $2 R / a$ ) MHD mode, were first detected in all large RFP's [69-74], and found to last for several energy confinement times. Later on, the SH states found in MHD simulations [29], triggered the discovery in the data base of RFX of similar states lasting over the whole flat top at about 1 MA [76]. The dominant magnetic mode has an amplitude 1 to few percents of the central magnetic field, and several times that of the other modes [75-77]. This motivates to qualify such regimes Quasi Single Helicity (QSH).

In contrast with MH plasmas, a "bean"-like hot structure is evident in SXR tomographic images in the QSH case in three large RFP's [75-79]. The "bean" structure is recovered when magnetic field lines are reconstructed from magnetic measurements, and turns out to correspond to a magnetic island. It comes with a decrease of magnetic chaos with respect to the $\mathrm{MH}$ case.

The local improvement of confinement due to magnetic flux surfaces in the plasma core shows up also in direct electron temperature profiles measurements performed by multipoint Thomson scattering [76, 78]. On top of the one found in the reversal region, a strong temperature gradient is found at the edges of the magnetic island: an internal transport barrier (ITB) is found to exist.

In three large RFP's there was a trend for an increased probability of the occurrence of QSH states when the current is increased [78-80].

\section{Upgrade of the RFX device}

Till the end of the 90's, the RFX device had a thick and distant conducting shell: vertical magnetic field penetration time $\tau_{\text {wall }}=450 \mathrm{~ms}$, shell to wall proximity $b / a \approx 1.24$. The discharge duration was about $100 \mathrm{~ms}$, with plasma currents up to $1 M A$. In the early 2000 s the machine was upgraded and renamed RFX-mod: a thin closer shell was installed and completely covered with 192 independently fed saddle coils ( 4 in the poloidal and 48 in the toroidal directions) for feedback control [81]: vertical magnetic field penetration time: $\tau_{\text {wall }}=50 \mathrm{~ms}$, shell to wall proximity: $b / a \approx 1.12$. The discharge duration is now up to $0.5 \mathrm{~s}$, with plasma currents up to the $2 \mathrm{MA}$ nominal value. Resistive wall modes can be completely avoided [82].

In this device, the RFP operation at currents $I \geq 0.6 M A$ is characterized preponderantly by QSH quasi-helical regimes. In fact, a QSH persistency up to $85 \%$ of the discharge flat top is obtained [83]. High current operation and high QSH persistency were made possible by the use and progressive optimization of the feedback control of the saddle coils aiming at creating a clean boundary analogous to an ideal virtual shell close to the plasma [7, 84, 85]. More recently, this clean boundary has included the possibility to excite a finite reference helicity [86, 87]. The standard high current operation shows the existence of time intervals with strong helical character, with duration and amplitudes growing with the plasma current $[85,88]$. The normalized amplitude of the dominant mode tends to become a constant $[83,88,89]$.

\section{From double to single magnetic axis}

During most of the duration of the flat top of RFX-mod high current discharges $(I \geq 1.5 M A)$, the plasma selforganizes into a helical state characterized by nested magnetic surfaces winding around a single helical axis, but enclosed in an almost axisymmetric boundary [68]. This state is the result of two successive bifurcations occurring when the current is progressively increased. The first one is of MHD type, and makes the plasma to leave the 


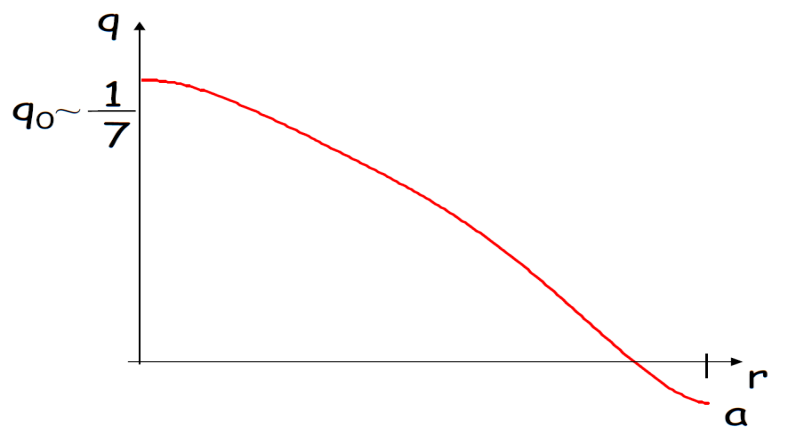

FIG. 7: q profile in a SHAx state of RFX-mod reconstructed by V3FIT-VMEC; courtesy by L. Marrelli and D. Terranova

Multiple Helicity $(\mathrm{MH})$ state, characterized by the presence of several resonant modes with similar amplitudes and to reach the Quasi Single Helicity (QSH) state which displays a single dominant mode and secondary ones with smaller amplitudes. At the lowest current intensities providing QSH states, the magnetic topology includes a magnetic island. Therefore the magnetic field displays two magnetic axes: the unperturbed axis-symmetric one and the one related to the island O-point. For such states, termed Double Axis (DAx) states, a thermal helical structure winding around the unperturbed magnetic axis is observed [91], characterized by an electron ITB [92, 93], and the maximum electron temperature (up to $1.2 \mathrm{keV}$ in 2011) is tightly correlated with the location of the magnetic island as reconstructed by external measurements. This ITB is the strongest in the rising phase of the QSH state [94].

The second bifurcation changes the topology of the magnetic field through the coalescence of the axis-symmetric O-point with the island X-point. Then the former island O-point becomes the only magnetic axis, which motivates to term Single Helical Axis (SHAx) this kind of QSH state [2, 3]. As explained in Sec. X A this topological bifurcation was first described theoretically. When broad electron temperature profiles were found in QSH states of RFX-mod, experimentalists confirmed immediately it was present [3]. The same phenomenology was found in MST [95, 96], and the DAx state was also found in EXTRAP T2R [97]. QSH states were also found in TPE-RX [98] and in RELAX [99, 100]. In RFX-mod there are also plasmas displaying external transport barriers close to the reversal surface [92].

In SHAx states, the region inside the above mentioned ITB has a flat temperature profile. It spans a significantly bigger volume than in DAx states [89], and the maximum electron temperature gradient at the ITB is a decreasing function of the amplitude of secondary modes [90]. Since this amplitude is reduced when plasma current is increased, it is expected that higher current plasmas will display even steeper thermal gradients and hotter helical cores. Plasma properties such as electron temperature, SXR emissivity and electron density have been found to be constant on helical magnetic surfaces [2] reconstructed with independent measurements, indicating that SHAx states are described by a MHD equilibrium characterized by almost invariant magnetic surfaces, in contrast with the low current MH states. The ITBs are located in the region where the magnetic shear profile of the helical QSH equilibria reverses [37, 68]. The improvement of the magnetic reconstructions with the V3FIT-VMEC code makes it even clearer now (see Fig. 7). In agreement with numerical simulations, providing proper helical boundary conditions to RFX-mod, strongly increases the persistence of the helical equilibrium [86]. As indicated by numerical simulations [40], it is even possible to excite a non resonant QSH $n=6$ state in RFX-mod by providing a corresponding helical boundary condition [87].

When a pellet is launched inside the hot domain, the density is found to peak there [101]. Nickel Laser Blow Off experiments show that impurities do not accumulate inside the helical structure [102]. The diffusion coefficient inside the island is of the same order as in $\mathrm{MH}$, while a radial domain characterized by an increased outward pinch is found at the ITB. High edge transport barriers have also been observed in the $q=0$ domain [68].

As yet the SHAx regimes are usually obtained at low densities $\left(n / n_{G}<0.35\right.$, with $n_{G}$ Greenwald density) [15]. The possibility of obtaining hot SHAx structures at higher densities relies on a more efficient fueling of the core by pellet injection accompanied by a better control of the recycling from the wall [10, 15]. A particle confinement time in SHAx states of $12 \mathrm{~ms}$ was obtained with pellet injection [101]. 


\section{ANALYTICAL DESCRIPTION OF THE SINGLE HELICITY RFP}

In 1958 RFP states were found by chance in the ZETA machine at Culham. In 1974, while computers were still unable to run a reasonable MHD simulation of the configuration, J.B. Taylor came with a relaxation theory [63] whose predictions had a fairly good agreement with experimental results till the late 90's. His work was the backbone of RFP theory till the emergence of the SH picture described in this chapter [4]. Now a whole series of experimental and theoretical results disagree with Taylor relaxation theory (see section 7 of [103] for more details).

We now describe the present status of the analytical calculation of the ohmic SH equilibrium magnetic field of RFP's, by using resistive MHD in cylindrical geometry and in the force-free limit. A large part of this section is a rephrasing of the analytical parts of Refs. [105] and [39].

\section{A. Helical Grad-Shafranov equation}

Let $(r, \theta, z)$ be the usual cylindrical coordinates, and $\hat{\mathbf{e}}_{x}=\nabla x$, with $x=r, \theta, z$ be the corresponding contravariant basis. The SH state of interest is defined by "toroidal" and "poloidal" mode numbers $n$ and $m$, such that all quantities depend on $\theta$ and $z$ only through $u=m \theta+k z$, where $k=-n / R$, and $2 \pi R$ is the axial periodicity. Let $\boldsymbol{\sigma}=\boldsymbol{\nabla} r \times \boldsymbol{\nabla} u=m \hat{\mathbf{e}}_{z} / r-k \hat{\mathbf{e}}_{\theta}$, and $f(r)=r /\left(m^{2}+k^{2} r^{2}\right)$. Calculations are simplified by introducing the auxiliary force-free magnetic field $\mathbf{h}=f(r) \boldsymbol{\sigma}$ (indeed it verifies $\boldsymbol{\nabla} \cdot \mathbf{h}=0$ and $\boldsymbol{\nabla} \times \mathbf{h}=\beta \mathbf{h}$ with $\beta=-2 m k /\left(m^{2}+k^{2} r^{2}\right)$ ). In a gauge where its radial component vanishes, the vector potential can be written as $\mathbf{A}=\psi \boldsymbol{\nabla} z+\Phi \boldsymbol{\nabla} \theta$, where $\psi$ and $\Phi$ are called poloidal and toroidal flux functions. This yields

$$
\mathbf{B}=\nabla \times \mathbf{A}=\nabla \psi \times \nabla z+\nabla \Phi \times \nabla \theta .
$$

A can be equivalently written as the sum of a component along $\nabla u$ and of $\chi \mathbf{h}$, where

$$
\chi=m \psi-k \Phi,
$$

is called helical flux. This yields

$$
\mathbf{B}=\nabla \chi \times \mathbf{h}+g \mathbf{h}
$$

where

$$
g=r \boldsymbol{\sigma} \cdot \mathbf{B}=m B_{z}-k r B_{\theta},
$$

is the helical magnetic field. Using equation (10) one finds that $\mathbf{B} \cdot \nabla \chi=0$, i.e., $\chi$ is a constant along magnetic field lines. Therefore such lines lie on magnetic surfaces. We call $S_{\chi}$ the surface with helical flux $\chi$, and we restrict to surfaces $S_{\chi}$ 's enclosing $r=0$, which is the case for the vicinity of the reversal surface we will focus on.

In Gaussian units equation (10) implies $\mathbf{J}=\boldsymbol{\nabla} \times \mathbf{B}=\left(\beta g-\triangle_{h} \chi\right) \mathbf{h}+\boldsymbol{\nabla} g \times \mathbf{h}$, when noticing that $\boldsymbol{\nabla} \times(\boldsymbol{\nabla} \chi \times \mathbf{h})=$ $-\triangle_{h} \chi \mathbf{h}$, where $\triangle_{h} \chi=f^{-1}\left[\partial_{r}\left(f \partial_{r} \chi\right)+r^{-1} \partial_{u}^{2} \chi\right]$; this is obtained by the successive estimates of the components of the vectors of interest. The force-free condition $\mathbf{J}=\lambda \mathbf{B}$ then requires $\boldsymbol{\nabla} g-\lambda \boldsymbol{\nabla} \chi$ to be colinear to $\mathbf{h}$. However, since $g$ and $\chi$ depend on $r$ and $u$ only, their gradients are in the plane defined by $\boldsymbol{\nabla} r$ and $\boldsymbol{\nabla} u$ which is orthogonal to $\mathbf{h}$. Therefore $\nabla g-\lambda \nabla \chi=0$, which implies equation

$$
g=g(\chi) \text { and } \mathrm{d} g / \mathrm{d} \chi=\lambda .
$$

Then the component parallel to $\mathbf{h}$ of the force-free condition implies the helical Grad-Shafranov equation [33, 104]

$$
\triangle_{h} \chi=g[\beta-\mathrm{d} g / \mathrm{d} \chi]
$$

where $\triangle_{h} \chi=f^{-1}\left[\partial_{r}\left(f \partial_{r} \chi\right)+r^{-1} \partial_{u}^{2} \chi\right]$, as shown in Appendix A of Ref. [105]. In the case $m=1$ and $n=0$ this is the classical equation for axis-symmetric plasmas.

\section{B. Parallel Ohm's law}

We describe the plasma by a general Ohm's law

$$
\mathbf{E}+\mathbf{v} \times \mathbf{B}=\eta \mathbf{J}+\frac{c_{1} \mathbf{J} \times \mathbf{B}-c_{2} \nabla p_{e}}{n e}
$$


where $\eta$ is the resistivity, and the $c_{i}$ 's are constants which may take on independently the values 0 or 1 depending on the type of Ohm's law of interest. If $c_{2}=1$ we restrict the electronic pressure $p_{e}$ to be constant along magnetic field lines and to be a fixed fraction of the total pressure. We consider the electric field to be stationary

$$
\mathbf{E}=E_{0} \hat{\mathbf{e}}_{z}-\nabla \phi
$$

where $E_{0}$ is the component related to the toroidal loop voltage of a real RFP. By performing the scalar product of Ohm's law with B, one obtains the parallel Ohm's law

$$
\text { B. } \nabla \phi=E_{0} \mathbf{B} \cdot \hat{\mathbf{e}}_{z}-\eta \mathbf{B} \cdot \mathbf{J} \text {. }
$$

We note that equations (16) and (14) do not depend on the $c_{i}$ 's, and therefore on the type of Ohm's law of interest. We obtain the average parallel Ohm's law

$$
\langle\mathbf{B} \cdot \boldsymbol{\nabla} \phi\rangle_{\chi}=E_{0}\left\langle B_{z}\right\rangle_{\chi}-\langle\eta \mathbf{B} \cdot \mathbf{J}\rangle_{\chi}=0
$$

by performing the flux surface average of equation (16) over $S_{\chi}$, the magnetic surface with flux $\chi$. This average is defined for a quantity $Q$ as

$$
\langle Q\rangle_{\chi}=\frac{\mathrm{d} I(Q)}{\mathrm{d} I(1)}
$$

where

$$
I(Q)=\int_{D(\chi)} Q \mathrm{~d}^{3} \mathbf{r},
$$

and $D(\chi)$ is the volume of axial length $2 \pi R$ within $S_{\chi}$. The derivation of equation (17) uses the force-free condition and relation $\langle\mathbf{B} \cdot \boldsymbol{\nabla} \phi\rangle_{\chi}=0$ resulting from the divergence theorem: $\int_{D(\chi)} \mathbf{B} \cdot \boldsymbol{\nabla} \phi d V=\int_{S_{\chi}} \phi \mathbf{B} \cdot \mathbf{d} \boldsymbol{\Sigma}=0$. The expression for $\langle Q\rangle_{\chi}$ is simpler when going from Cartesian coordinates to $(\chi, u, \theta)$. The corresponding Jacobian $J$ is given by

$$
J^{-1}=\nabla \chi \times \nabla \theta \cdot \nabla u=\frac{k}{r} \partial_{r} \chi
$$

Then

$$
\langle Q\rangle=\langle J Q\rangle_{0} /\langle J\rangle_{0}
$$

where the average $\langle F\rangle_{0}$ of a quantity $F(\chi, u)$ is defined by

$$
\langle F(\chi, u)\rangle_{0}=\frac{1}{2 \pi} \oint F(\chi, u) \mathrm{d} u .
$$

If the force-free condition $\mathbf{J}=\lambda \mathbf{B}$ is satisfied, the average parallel Ohm's law becomes

$$
E_{0}\left\langle B_{z}\right\rangle_{\chi}=\lambda\left\langle\eta \mathrm{B}^{2}\right\rangle_{\chi}
$$

\section{Pinch-stellarator equation}

Here SH ohmic RFP states are described analytically as small helical perturbations of axis-symmetric ohmic pinches with small edge conductivity and axial magnetic field, called ultimate pinches. At this point one might think about applying directly perturbation theory to Eqs. 13 and 23, taking an ultimate pinch as zeroth order equilibrium, with the perturbation amplitude of the asymmetric magnetic flux as a small parameter of the ordering. However, the calculation must be done to second order, and the analytical calculation of the solution of the corresponding differential equations looks formidable. Fortunately interesting new physics may be found without computing the full magnetic field, but only $\left\langle B_{z}\right\rangle_{\chi}$ to second order. Indeed the safety factor of flux surface $S_{\chi}$ is proportional to $\left\langle B_{z}\right\rangle_{\chi}$. This is readily shown by noting that the "poloidal" (azimuthal) flux $F_{\text {pol }}(\chi)$ enclosed between $S_{\chi}$ and the perturbed magnetic axis is the volume integral of $B_{\theta} / r$ divided by $2 \pi$. Similarly the "toroidal" (axial) flux $F_{\text {tor }}(\chi)$ inside $S_{\chi}$ is 
the volume integral of $B_{z} / R$ divided by $2 \pi$. Using the definition (18) of the surface average, this implies the safety factor is

$$
q(\bar{\psi}) \equiv \mathrm{d} F_{\text {tor }} / \mathrm{d} F_{\text {pol }}=\frac{\left\langle B_{z}\right\rangle_{\chi}}{R\left\langle B_{\theta} / r\right\rangle_{\bar{\psi}}}
$$

The reversal parameter of a RFP is classically defined as $F=B_{z}^{0,0}(a) /\left\langle B_{z}\right\rangle_{v}$ where \langle\rangle$_{v}$ means "volume average inside $r=a$ ". We further notice that, whenever the plasma is bounded by an ideal shell, at the shell radius $r=a$ a magnetic surface must be cylindrical. Therefore a negative value of $F$ means a negative value of $\left\langle B_{z}\right\rangle$ and of $q$ at the wall.

In order to compute $\left\langle B_{z}\right\rangle_{\chi}$ to second order, we follow first closely Ref. [33]. Using equation (12) for the helical magnetic field $g$, we average equation (11) defining $g$ over $S_{\chi}$, we take its derivative over $\chi$, and we use Ampere's law and the force-free relation. In the following it will be useful to parameterize flux surfaces by $\bar{\psi}(\chi)=\langle\psi(\chi, u)\rangle_{0}$. Various steps given in Appendix C of Ref. [105], yield the pinch-stellarator equation

$$
\frac{\mathrm{d}}{\mathrm{d} \bar{\psi}}\left\langle B_{z}\right\rangle_{\bar{\psi}}=\frac{E_{0}}{\left\langle\eta \mathrm{B}^{2}\right\rangle_{\bar{\psi}}}\left\langle B_{z}\right\rangle_{\bar{\psi}}+S(\bar{\psi})
$$

with

$$
S(\bar{\psi})=k\left\langle\left(\partial_{\bar{\psi}} r\right) \partial_{u} B_{r}\right\rangle_{0}+(k / m) \mathrm{d}\left(\left\langle r B_{\theta}\right\rangle-\left\langle r B_{\theta}\right\rangle_{0}\right) / \mathrm{d} \bar{\psi} .
$$

Equation (25) is a slightly different version of equation (34) of Ref. [33] which was a new formulation of an equation already derived in Ref. [106]. Both references make the following remarks: (i) $S(\bar{\psi})$ vanishes for a pinch with axial symmetry; (ii) equation $(25)$ is a first order linear ordinary differential equation for $\left\langle B_{z}\right\rangle_{\bar{\psi}}$, which may be formally integrated; (iii) for an axially symmetric pinch it holds for all radii, and implies that $\left\langle B_{z}\right\rangle_{\bar{\psi}}$ cannot reverse at the plasma edge, confirming the statement of sec. IX, but may be small there; (iv) in a stellarator since $E_{0}=0, S(\bar{\psi})$ is the only term providing a radial variation of $\left\langle B_{z}\right\rangle_{\bar{\psi}}$; (v) since according to Eq. (8) $\bar{\psi}$ decreases from the core to the edge, for a positive $\left\langle B_{z}\right\rangle_{\bar{\psi}}$ in the center, a positive $S(\bar{\psi})$ in a finite edge radial domain in $\bar{\psi}$ may a priori provide a reversal of $\left\langle B_{z}\right\rangle_{\bar{\psi}}$ in that domain. Using the equation (10) of Ref. [106], which is the analogue of the present equation (25), Ref. [107] proved numerically the existence of reversal for a pinch with twisted elliptical flux surfaces. The tokamak corresponds to $S(\bar{\psi})=0$, and to $E_{0}$ small enough to provide a radially almost constant axial magnetic field.

It is interesting to note that the experimental profiles of the reversed axis-symmetric part of the toroidal field in RFP's are rather flat in the reversal domain after a steady decrease in the non reversed one (see Fig. 2 of [108]). This is in harmony with the leading term going from the pinch one to stellarator one in the reversal domain. In this domain these experimental profiles are rather shallow with respect to what Taylor relaxation theory [63] predicts with the Bessel function model: $B_{z} \sim \mathbf{J}_{0}(\mu r)$ with $\mu$ a constant. In such a model a finite edge poloidal current is necessary to provide reversal, and this was thought to be the origin of the high $V_{\text {loop }}$ in RFP's. In contrast the pinch-stellarator equation shows that no current is necessary in the highly resistive edge of a RFP to provide the reversal. This indicates that the decrease of the experimental $V_{\text {loop }}$ in RFP's might be obtained by a mere decrease of the resistivity inside the ITB, which might result from operation at higher currents [90].

\section{Single helicity ohmic RFP states}

If the resistivity is high enough in a finite radial domain $D=[r *, a]$ enclosing the plasma edge $r=a$, the pinchstellarator equation (25) shows that for the unperturbed ultimate pinch, $B_{z}$ stays small in $D$ if it is small at $r=a$. Then parallel Ohm's law (23) shows that $\lambda$ is also small in $D$. Therefore, due to equation (12) for the helical magnetic field $g$, up to a correction of order $B_{z}(a), g(\chi)$ is a constant $g_{0}$ in $D ; g_{0}$ turns out to be positive since the axial current is positive, and since $k<0$. From now on, for any quantity $Q, Q^{\prime}$ means $\partial_{r} Q$, or $\mathrm{d} Q / \mathrm{d} r$ if $Q$ depends on $r$ only. Then the helical Grad-Shafranov equation (13) can be readily integrated in $D$ to give

$$
\chi_{0}^{\prime}=m g_{0} / k r,
$$

up to a correction of order $B_{z}(a)$, by taking into account that $\lambda$ is small in $D$, that the axial current and $r B_{\theta}$ are almost constant in $D$, and that equation (10) defining $\mathbf{B}$ in terms of $\chi$ and $g$ implies

$$
\partial_{r} \chi=-r \nabla u \cdot \mathbf{B} \text {. }
$$

We now perturb this ultimate pinch with a small $(m, n)$ perturbation that is non resonant in $D$. Let $\epsilon \chi_{1}(r) \cos (u)$, with $\epsilon$ small, be the corresponding perturbation of $\chi_{0}(r)$. We describe $\chi_{1}(r)$ by the Newcomb equation obtained 
by linearization of the helical Grad-Shafranov equation (13) without any inhomogeneous term; this is natural if we consider the helical state to result from a tearing-like instability of the ultimate pinch. Newcomb equation is

$$
\frac{\mathrm{d}}{\mathrm{d} r}\left(f \frac{\mathrm{d} \chi_{1}}{\mathrm{~d} r}\right)-\frac{1}{r} \chi_{1}=\left[\beta(r) \lambda-\lambda^{2}-g \frac{\mathrm{d} \lambda}{\mathrm{d} \chi}\right] \chi_{1}
$$

where all functions of $\chi$ are evaluated at $\chi_{0}(r)$. Since $g$ is assumed to be constant in $D$ to zeroth order, the right hand side of Eq. (29) vanishes in this interval, and we are left with

$$
\frac{\mathrm{d}}{\mathrm{d} r}\left(f \frac{\mathrm{d} \chi_{1}}{\mathrm{~d} r}\right)-\frac{1}{r} \chi_{1}=0
$$

inside $D$. There it is convenient to introduce the flux label $\rho$ such that $\chi=\chi_{0}(\rho)$. Since the range of $\chi$ may be larger than that of $\chi_{0}$, we complement $\chi_{0}(r)$ for $r>a$ by a straight line tangent to $\chi_{0}(r)$ at $r=a$. Then on the flux surface labeled by $\rho, r$ is given by

$$
r(\rho, u)=\rho+\epsilon r_{1}(\rho) \cos u+O\left(\epsilon^{2}\right)
$$

where $r_{1}(\rho)=-\chi_{1}(\rho) / \chi_{0}^{\prime}(\rho)$; the $O\left(\epsilon^{2}\right)$ terms contribute only at order higher than 2 in the following results. As shown in Appendix D of Ref. [105], with the above definitions $S(\rho)$ can be readily computed

$$
S(\rho)=\frac{\epsilon^{2} k^{2}[m-n q(\rho)]}{m g_{0} \rho \chi_{0}^{\prime}(\rho)} P(\rho)
$$

where

$$
P(\rho)=f^{2}\left(m^{2}+2 k^{2} \rho^{2}\right)\left(\chi_{1}^{\prime}\right)^{2}+2 \rho \chi_{1}^{\prime} \chi_{1}+\chi_{1}^{2} .
$$

and $q(\rho)$ is the safety factor defined as follows. Using equation (8) the "poloidal" (azimuthal) flux enclosed between $S_{\bar{\psi}}$ and the perturbed magnetic axis (labeled by $\bar{\psi}_{0}$ ) of the ultimate pinch is readily computed to be $F_{\text {pol }}=-2 \pi R\left[\bar{\psi}-\bar{\psi}_{0}\right]$. Similarly the "toroidal" (axial) flux inside $S_{\bar{\psi}}$ is $F_{\text {tor }}=2 \pi \bar{\Phi}(\bar{\psi})$ where $\bar{\Phi}(\bar{\psi})=\langle\Phi(\bar{\psi}, u)\rangle_{0}$. As a result the safety factor is

$$
q(\bar{\psi}) \equiv \mathrm{d} F_{\text {tor }} / \mathrm{d} F_{\text {pol }}=-R^{-1} \mathrm{~d} \bar{\Phi} / \mathrm{d} \bar{\psi} .
$$

Define the normalized logarithmic derivative of $\chi_{1}, L=a \chi_{1}^{\prime} / \chi_{1}$. Then for $S(\rho)$ to be positive $P(\rho) / \chi_{1}^{2}$, a quadratic polynomial in $L$, must be negative. Therefore a necessary criterion for reversal $([105])$ is that $L(\rho)$ must be in between the two roots of this polynomial which are both negative,

$$
L_{ \pm}=-\frac{a\left(1+k^{2} \rho^{2}\right)}{\rho\left[1+(1 \pm 1) k^{2} \rho^{2}\right]},
$$

where we made $m=1$ for simplicity, and having in mind this corresponds to the most unstable modes in RFP's.

The criterion is suggestive that a finite edge radial magnetic field might be favorable for field reversal. In accordance with this, visco-resistive MHD simulations show that helical equilibria with low perturbation amplitudes achieve reversal when a finite edge radial magnetic field is applied [105]. Numerical simulations show that the criterion works for large perturbations of the pinch too, in particular those leading to states with a single helical axis. The necessary criterion is found to be satisfied in the RFX-mod experiment for reversed states with non zero reference edge radial magnetic field [105]. These experimental and numerical results show that the validity of the criterion is more general than suggested by the perturbative approach used for its derivation: nonlinear corrections look weak, and the condition that the edge temperature is much smaller than the central one sounds as a leading factor. Helical boundary conditions for the radial magnetic field, mimicking a helical modulation of the plasma magnetic boundary similar to the experimental one, are found to play an essential role for the qualitative and quantitative agreement of MHD simulations with respect to experimental observations in both reversed-field pinch (RFP) and tokamak magnetic configurations $[40,66,67]$.

We now show how Eqs. (25), (26), and (32) can be used to exhibit SH ohmic RFP states. If $L_{-}(a)<L(a)<L_{+}(a)$, there is a finite domain $\left[r_{S}, a\right]$ where $S>0$. Consider an ultimate pinch with a high resistivity in this domain, and such that $B_{z}\left(r_{S}\right) / B_{z}(0)=\mathrm{O}\left(\epsilon^{2+s}\right)$ with $s>0$. It may be for instance a Bessel function model (BFM) [109] where $r_{S}$ is close enough to the value of $\rho$ where the zeroth order Bessel function vanishes (the corresponding resistivity profile is obtained by solving Eq. (23) for $\eta$ ). When computing directly $\left\langle B_{z}\right\rangle$ by using the second order expressions for $\chi, \lambda$ and $g$, an appropriate choice of the integration constants occurring at second order enables to set $\left\langle B_{z}\right\rangle_{\rho=r_{S}}=B_{z}\left(r_{S}\right)$. 
Then in $\left[r_{S}, a\right]$ the r.h.s. of Eq. $(25)$ is $\mathrm{O}\left(\epsilon^{2}\right)<0$ for $\epsilon$ small. Integrating this equation from $r_{S}$ to $a$ yields $\left\langle B_{z}\right\rangle_{\rho=a}=\mathrm{O}\left(\epsilon^{2}\right)<0$ for $\epsilon$ small. Therefore there is reversal of $\left\langle B_{z}\right\rangle$ at the edge provided the system can settle to a finite and small $\epsilon$. For a tearing mode resonating inside the plasma, this is controlled by the stability index $\Delta^{\prime}$ which depends on $L(a)$. In a cylindrical full MHD model $\Delta^{\prime} \sim \sqrt{\epsilon} \ln (\epsilon)$ [110]. For the simple case where the ultimate pinch is a BFM inside of $r_{S}, \Delta^{\prime}(L(a))$ can be analytically computed by an extension of the calculation in Ref. [111]. This yields a formula for $\Delta^{\prime}(\mu, L(a))$ where $\mu$ is the constant value of $\lambda$ for the BFM. Since $S(\rho)$ has a weak dependence on $\mu$ through $\chi_{0}^{\prime}(\rho)$, it is possible to adjust $\mu$ such that $\Delta^{\prime}(\mu, L(a))$ be small and provide a finite and small $\epsilon$ at saturation of the tearing mode. Therefore for this class of ultimate pinches the existence of perturbed SH ohmic RFP states is proved. For more general ultimate pinches one should resort to numerical calculations, but by continuity with the BFM case, one should expect a broader class of ultimate pinches to provide RFP states with a finite and small $\epsilon$.

\section{E. Calculation of the dynamo}

Equation (8) implies that

$$
\mathbf{B} \cdot \boldsymbol{\nabla} u=-(k J)^{-1} .
$$

Writing

$$
\nabla \phi=\partial_{u} \phi \nabla u+\partial_{\chi} \phi \nabla \chi
$$

the last expression in equation (8) yields

$$
\mathbf{B} \cdot \boldsymbol{\nabla} \phi=-\partial_{u} \phi(k J)^{-1},
$$

Equations (16) and (38) finally yield

$$
\partial_{u} \phi=-r \partial_{\chi} r E_{0} \mathbf{B} \cdot \hat{\mathbf{e}}_{z}-\eta \mathbf{B} \cdot \mathbf{J},
$$

where all quantities are computed at the position defined by $(\chi, u)$. This equation is thoroughly discussed in Sec. 2.1 .2 of [39].

Equations (14) and (15) imply that the perpendicular component of the velocity can be written as

$$
v_{\perp}=v_{\perp e s}+v_{\perp n e s}
$$

where the electrostatic component $v_{\perp e s}$ is given by Eq. 6 and

$$
v_{\perp n e s}=\left[E_{0} \hat{\mathbf{e}}_{z}-\eta \mathbf{J}-\frac{c_{1} \mathbf{J} \times \mathbf{B}-c_{2} \boldsymbol{\nabla} p_{e}}{n e}\right] \times \frac{\mathbf{B}}{\mathrm{B}^{2}},
$$

is the non electrostatic component of $v_{\perp}$. These equations show that the dynamo velocity field is slaved to the magnetic equilibrium.

Reference [39] shows that $v_{\perp e s}$ may be written as

$$
v_{\perp e s}=-\partial_{u} \phi \nabla u \times \frac{\mathbf{B}}{\mathrm{B}^{2}}-\partial_{\chi} \phi \nabla \chi \times \frac{\mathbf{B}}{\mathrm{B}^{2}} .
$$

By decomposing the estimate of $v_{\perp e s}$ over $\nabla u \times \mathbf{B} / \mathrm{B}^{2}$ and $\nabla \chi \times \mathbf{B} / \mathrm{B}^{2}$, one can estimate both $\partial_{u} \phi$ and $\partial_{\chi} \phi$. The first one may be compared to its estimate provided by equation (39). The combination of both expressions provides an estimate of $\phi(\chi, u)$. Then taking the Laplacian of $\phi$ yields the charge density due to the ambipolarity constraint.

The importance of the electrostatic field for the RFP dynamo shows up when considering the spatial mean, over $\theta$ and $z$, of the parallel Ohm's law 16

$$
\langle\eta \mathbf{B} \cdot \mathbf{J}\rangle=E_{0}\left\langle B_{z}\right\rangle-\langle\mathbf{B} \cdot \nabla \phi\rangle
$$

The radial profiles of the terms in this equation in a $\mathrm{SH}$ simulation of the RFP are displayed in Fig. 5a of [112]. It shows the contribution from the induction electromotive force proportional to $E_{0}$ turns out to be larger than the one from the mean parallel current density in the plasma core, and smaller in the edge. The difference is balanced by the electrostatic term, which provides what are often named the anti-dynamo contribution in the core and direct dynamo contribution in the edge. It is worth noticing that there is no contribution from the dynamo term $\mathbf{v} \times \mathbf{B}$ in this case, since we are considering the scalar product of Ohm's law with the total magnetic field. 
The standard picture where this dynamo term is important is recovered when taking the scalar product of Ohm's law with the mean axis-symmetric magnetic field

$$
\left\langle\eta \mathbf{B}_{0} \cdot \mathbf{J}\right\rangle=E_{0}\left\langle\eta \mathbf{B}_{0} \cdot \hat{\mathbf{e}}_{z}\right\rangle-\left\langle\mathbf{B}_{0} \cdot \mathbf{v} \times \mathbf{B}\right\rangle,
$$

as shown in Fig. 5b of [112]. In this case, instead of the contribution from the electrostatic field (which vanishes since $\boldsymbol{\nabla} \phi$ has no axis-symmetric component) the dynamo/anti-dynamo action is provided by the usual $\left\langle\mathbf{B}_{0} \cdot \mathbf{v} \times \mathbf{B}\right\rangle$ term. In reality the average in 44 is generally done over the toroidal and poloidal angles. Therefore both the standard and the "electrostatic" picture are equivalent. However the former has been unable to explain the origin of the velocity field, while the latter does. The "electrostatic" picture applies as well to the nonlinear tearing mode which has a dynamo too [113]. Both this saturated mode and the RFP SH ohmic state belong in the same family of stationary ohmic states.

Finally it is worth noting that the helical potential generating the dynamo velocity field might bring an experimental issue. Indeed if the plasma is surrounded by a toroidally symmetric conducting wall, while the electrostatic equipotential surfaces are helical, then radial electrical currents are driven at the wall, which perturb both the local magnetic field and the electrostatic potential.

\section{CONCLUSION}

This chapter defined the RFP, presented the historical evolution of magnetic self-organization of these machines, and provided the present theoretical picture about the configuration. The latter is now recalled. The current-carrying wire model showed how a kink unstable system inside a flux conserver may be nonlinearly stabilized by field reversal. The RFP turns out to use a quite similar way to self-organize, as shown by MHD simulations. The latter proved the ohmic constraint to be of paramount importance for the RFP. This constraint requires in particular the magnetic field of an ohmic RFP to be helically distorted. This helical deformation is provided naturally by the nonlinear saturation of a resistive kink instability. This nonlinear feature of the RFP configuration prevents the occurrence of disruptions. It comes with a helical electrostatic electric field driving a drift which provides the main contribution to the dynamo velocity field, as occurs for the nonlinear tearing mode.

From both its magnetic and velocity fields, the RFP may be viewed as a helically distorted paramagnetic pinch: it is a helical self-organized ohmic magnetic configuration. The RFP may be in two different states which are continuously connected by changing dissipation: a single helicity ( $\mathrm{SH}$ ) and a multiple helicity (MH) state. In the SH state the helical deformation of the plasma is uniformly distributed along the plasma, and magnetic field lines are regular. In the $\mathrm{MH}$ state the helical deformation of the plasma is localized and magnetic field lines are chaotic. The helical shape of the SH plasmas introduces analogies with the stellarator [16]. However the helical distortion decreases when going close to the shell, which alleviates the issue of fast particle confinement. The main theoretical challenge now is the introduction of self-consistency in the resistivity profile as a result of heat transport, which rules the electron temperature profile. This new element of self-organization will be addressed with the PIXIE3D nonlinear MHD initial value code [114], recently benchmarked in tokamak and RFP contexts with the SpeCyl code [115]. The feedback of heat transport on MHD states is not trivial at all, since it was recently shown to modify the stability of pressure-driven resistive g-modes [116].

Experimental results in all present or recent RFP's confirm many of the features of the present theoretical picture. In RFX-mod at high plasma currents (above $1 \mathrm{MA}$ ) there is a new regime, where the plasma spontaneously selforganizes in a single-axis helical state, with an internal transport barrier. This regime comes close to the theoretically predicted chaos-free helical ohmic equilibrium with a single magnetic axis. Experiments clearly indicate that the plasma is naturally choosing this improved regime at high current if a clean magnetic boundary condition is provided. The reason why high current is beneficial is still awaiting a theoretical explanation. The main experimental challenge in RFX-mod is presently to reach higher particle densities by pellet injection and by reduction of wall recycling. This is a prerequisite for a meaningful scaling of energy confinement time with plasma parameters.

Present experimental and theoretical results prove a change of paradigm occurred for the RFP: this configuration is no longer dominated by magnetic turbulence and chaos. From a theoretical viewpoint this new paradigm stems from the existence of two bifurcations: that from multiple to single helicity, and that from a double to a single helical axis. This new paradigm comes with other good news. First, no poloidal currents need to be driven in the resistive edge of the plasma. Second, the dynamo mechanism is no longer mysterious: it is a mere electrostatic drift due to the helical deformation of the current lines, as occurs for the nonlinear tearing mode. The RFP used to be a terrible confinement configuration: might it become a terrific one?

The RFP comes with interesting transversal physics involving the other magnetic configurations. Analogies have already been found between the tokamak and the RFP as long as edge physics [117], the Greenwald limit [15, 118], and ITB's [68] are concerned. Furthermore the RFP is an excellent test bed for the efficient control of multiple resistive 
wall modes [14, 82]. Issues like ITB's or the impact of non axis-symmetry on confinement might provide synergies with the stellarator studies. As a result the RFP is also useful to bring support to the present two main lines of magnetic confinement.

I thank D. Bonfiglio, S. Cappello, G. Marchiori, and F. Sattin for useful comments, and P. Ghendrih for his help in editing the document.

[1] Chapman B E et al. 2001 Phys. Rev. Lett. 87205001

[2] R. Lorenzini et al., Nature Phys. 5, 570 (2009)

[3] R. Lorenzini et al. Phys. Rev. Lett. 101, 025005 (2008)

[4] Ortolani S and Schnack D D 1993 Magnetohydrodynamics of Plasma Relaxation (Singapore: World Scientific)

[5] J.A. Wesson, Tokamaks, (Oxford University press, Oxford, 2004)

[6] P. Martin et al., 2011 Nucl. Fusion 51094023

[7] P. Zanca et al. Phys. Plasmas 20, 056112 (2013)

[8] Rostagni G 1995 Fus. Eng. Design 25301

[9] M. Greenwald, Plasma Phys. Contr. Fus. 44, R27 (2002)

[10] P. Piovesan, et al., Phys. Plasmas 20, 056112 (2013)

[11] Monchaux et al., Phys. Fluids 21, 035108 (2009)

[12] M. Gobbin, G. Spizzo, L. Marrelli and R. B. White, Phys. Rev. Lett. 105, 195006 (2010)

[13] P. Zanca et al. Plasma Phys. Contr. Fus. 51, 015006 (2009)

[14] P. Zanca et al. Plasma Phys. Contr. Fus. 54, 124018 (2012)

[15] M. E. Puiatti, to be published in Plasma Phys. Control. Fusion

[16] M. Gobbin, et al., Phys. Plasmas 18, 062505 (2011)

[17] McCollam K. J., Anderson J. K., Blair A. P., et al., Phys. Plasmas 17, 082506 (2010)

[18] Dahlin J.-E., Scheffel J., Nucl. Fusion 47, 1184 (2007)

[19] B.E. Chapman, Nucl. Fusion 49, 104020 (2009)

[20] Lawson J. D., Proc. Phys. Soc. B70, 6 (1957)

[21] Escande D E and Bénisti D 1997 in Proc. of the 7th European Fusion Theory Conference ed A Rogister (Jülich: Forschungzentrum KFA) 1127

[22] Escande D F et al. 2000 Plasma Phys. Contr. Fus. 42 B243

[23] Kadomtsev B B 1992 Tokamak plasma: a complex physical system, (Bristol: IOP) p 34

[24] Antoni V et al., 1994 in Proc. 15th IAEA Int. Conf. on Plasma Phys. and Contr. Nucl. Fus. Res.,Sevilla) 2405

[25] D. Bonfiglio, S. Cappello, and D. F. Escande, Phys. Rev. Lett. 94, 145001 (2005).

[26] Cowling T G 1957 Magnetohydrodynamics, (New York: Interscience)

[27] D. Bonfiglio, S. Cappello, R. Piovan, L. Zanotto and M. Zuin, Nucl. Fusion 48 (2008) 115010

[28] Cappello S and Biskamp D 1996 Nucl. Fusion 36571

[29] Cappello S and Escande D F 2000 Phys. Rev. Lett. 853838

[30] Sovinec C R et al. 2001 Phys. Plasmas, 8475

[31] Cappello S and Paccagnella R 1990 in Proc. Workshop on Theory of Fusion Plasmas, ed E Sindoni (Bologna: Compositori) p 595

[32] Cappello S and Paccagnella R 1992 Phys. Fluids B 4611

[33] Finn J M Nebel R A and Bathke C C 1992 Phys. Fluids B4 1262

[34] S. Cappello, Plasma Phys. Contr. Fus. 46, B313 (2004)

[35] D.F. Escande et al., Phys. Rev. Lett. 85, 1662 (2000)

[36] Sovinec T A, et al. 2003 Phys. Plasmas 101727

[37] Gobbin M. et al., Phys. Rev. Lett. 106025001

[38] Bonfiglio D., Veranda M., et al., in Proc. of Joint Varenna-Lausanne Workshop on the Theory of Fusion Plasmas 2010 eds. Garbet X, Sauter O ( Varenna, Italy) 260012003 (2010)

[39] S. Cappello et al., Nucl. Fusion 51, 103012 (2011).

[40] M. Veranda et al., Plasma Phys. Contr. Fus. 55, 074015 (2013).

[41] Caramana E J, Nebel R A and Schnack D D 1983 Phys. Fluids 261305

[42] Aydemir A Y and Barnes D C 1984 Phys. Rev. Lett. 52930

[43] Holmes J A et al. 1985 Phys. Fluids 28261

[44] Schnack D D et al. 1985 Phys. Fluids 28321

[45] Kusano K and Sato T 1986 Nucl. Fusion 261051

[46] Sykes A and Wesson J 1977 Proceedings of the 8th EPS Conference on Controlled Fusion and Plasma Physics, Prague 1 80 (European Physical Society)

[47] Montgomery D 1992 Plasma Phys. Control. Fusion 341157

[48] Montgomery D 1993 Plasma Phys. Control. Fusion 35 B105

[49] Tebaldi C and Ottaviani M 1999 J. Plasma Phys. 62513 
[50] D. Grasso, R. J. Hastie, F. Porcelli, and C. Tebaldi, Phys. Plasmas 15, 072113 (2008)

[51] Bonfiglio D, Cappello S and Escande D F 2004 Charge Separation as the basis of the Reversed Field Pinch dynamo, RFP 10th IEA/RFP-Workshop, Padova, Italy,

http://www.igi.pd.cnr.it/wwwevent/RFPWS/RFPWS_presentations.html

[52] D. Bonfiglio et al. Theory of Fusion Plasmas, AIP conference proceedings 8713 (2006)

[53] M. Onofri, F. Malara, and P. Veltri, Phys. Rev. Lett. 101, 255002 (2008)

[54] Kusano K and Sato T 1990 Nucl. Fusion 302075

[55] Terranova D et al. 2000 Plasma Phys. Control. Fusion 42843

[56] Biskamp D D 1993 Nonlinear Magnetohydrodynamics, (Cambridge: Cambridge University Press)

[57] Scheffel J and Schnack D D 2000 Nucl. Fusion 401885

[58] Cappello S, D'Angelo F, Escande D F, Paccagnella R, and Bénisti D 1999 Proc. 26th EPS Conference on Controlled Fusion and Plasma Physics, Maastricht (European Physical Society) 23J 981

[59] Escande D F, Paccagnella R et al. 2000 Phys. Rev. Lett. 853169

[60] G. Spizzo, S. Cappello, A. Cravotta, D. Escande, I. Predebon, L. Marrelli, P. Martin, and R. White, Phys. Rev. Lett. 96, 025001 (2006).

[61] M. G. Rusbridge, Plasma Phys. Contr. Fus. 33, 1381 (1991)

[62] S. Cappello and D. Biskamp, Proc. International Conf. on Plasma Phys. Nagoya, 1996

[63] Taylor J B 1974 Phys. Rev. Lett. 331139

[64] Nebel R A, Finn J M and Bathke C C 2004 Single helicity and quasi-single helicity states in reversed field pinches, RFP 10th IEA/RFP-Workshop, Padova, Italy,

http://www.igi.pd.cnr.it/wwwevent/RFPWS/RFPWS_presentations.html

[65] Bodin H A B 1990 Nucl. Fusion 301717

[66] D. Bonfiglio, M. Veranda et al., Phys. Rev. Lett. 111, 085002 (2013)

[67] D. Bonfiglio et al., Proceeding of the 40th EPS Conference on Plasma Physics (2013), P2.145.

[68] M. E. Puiatti, Plasma Phys. Control. Fusion 51, 124031 (2009)

[69] Brunsell P R 1993 Phys. Fluids B5 885

[70] Nordlund P and Mazur S 1994 Phys. Plasmas 14032

[71] Hirano Y, Yagi Y et al. 1997 Plasma Phys. Contr. Fus. 39 A393

[72] Martini S et al. 1999 Plasma Phys. Contr. Fus. 41 A315

[73] Sarff, J S et al. 1997 Phys. Rev. Lett. 7862

[74] Martin P 1999 Plasma Phys. Contr. Fus. 41 A247

[75] Piovesan P, Spizzo G, Yagi Y et al. 2004 Phys. Plasmas, 11151

[76] Escande D F, Martin P., Ortolani S et al. 2000 Phys. Rev. Lett. 851662

[77] Martin P et al. 2000 Phys. Plasmas, 71984

[78] Martin P et al. 2003 Nucl. Fusion 431855

[79] Marrelli L et al. 2002 Phys. Plasmas 92868

[80] Bolzonella T and Terranova D 2002 Plasma Phys. Control. Fusion 442569

[81] P. Sonato et al. Fusion Eng. Des. 66, 161 (2003)

[82] T. Bolzonella, Fusion Eng. Des. 82, 1064 (2007)

[83] P. Piovesan et al., in proc. 35th EPS Conf. on Plasma Physics, Herssonissos, Crete O4.029 (2008)

[84] P. Zanca et al. Nucl. Fusion 47, 1425 (2007)

[85] L. Marrelli et al., Plasma Phys. Contr. Fus. 49, B359 (2007)

[86] P. Piovesan, et al., Plasma Phys. Control. Fusion 53, 084005 (2011)

[87] S. Cappello et al., in Proceedings of the 24th IAEA Fusion Energy Conference, San Diego, USA (2012) http://wwwnaweb.iaea.org/napc/physics/FEC/FEC2012/papers/199_THP216.pdf.

[88] P. Piovesan, M. Zuin, A. Alfier et al., Nucl. Fus. 49, 085036 (2009)

[89] M. Valisa et al., Plasma Phys. Contr. Fus. 50, 124031 (2008)

[90] R. Lorenzini et al., Nucl. Fusion 52, 062004 (2012)

[91] P. Franz et al. Phys. Plasmas 13, 012510 (2006)

[92] M. E. Puiatti, M. Valisa, et al., Nucl. Fusion 51, 073038 (2011)

[93] A. Alfier et al. Plasma Phys. Contr. Fus. 50, 035013 (2008)

[94] P. Franz, M. Gobbin, L. Marrelli, A. Ruzzon, et al., Nucl. Fusion 53, 053011 (2013)

[95] W.F. Bergerson, et al., Phys. Rev. Lett. 107, 255001 (2011)

[96] F. Auriemma, P. Zanca, et al., Plasma Phys. Contr. Fus. 53, 105006 (2011)

[97] L. Frassinetti, et al., Phys. Plasmas 14, 112510 (2007)

[98] Hirano Y, et al., Phys. Plasmas 12112501 (2005)

[99] Masamune S., Sanpei A., et al., J. Fusion Energ. 28, 187 (2009)

[100] Ikezoe R, Masamune S, Oki K, et al., J. Phys. Soc. Jpn. 81, 115001 (2012)

[101] D. Terranova et al., Nucl. Fusion 50, 035006 (2010)

[102] S. Menmuir et al., Proc. 36th EPS Conf. on Plasma Phys. Sofia, 2009

[103] S. Cappello, Theory of Fusion Plasmas, Joint Varenna-Lausanne International Workshop, AIP Conf. Proc. 1069, 27-39 (2008)

[104] Johnson J.L. et al. 1958 Phys. Fluids 1281

[105] D. Bonfiglio, D. F. Escande, P. Zanca, and S. Cappello, Nucl. Fusion 51, 063016 (2011) 
[106] Pustovitov V.D. 1982 Pis'ma Zh. Eksp. Teor. Fiz. 353 [1982 JETP Lett. 35 1]

[107] Vabishchevich P N et al. 1983 Fiz. Plazmy 9484 transl. Soviet J. Plasma Phys. JETP 9280

[108] H.A.B. Bodin, IAEA Fusion Energy Conference, Lausanne, vol. 1, p. 417 (1984)

[109] S. Lundquist, Phys. Rev. 83, 307 (1951).

[110] N. Arcis, D. F. Escande, and M. Ottaviani, Phys. Plasmas, 14, 032308 (2007).

[111] R.D. Gibson, K.J. Whiteman, Plasma Phys. 10, 1101 (1968).

[112] Cappello, D. Bonfiglio, and D. F. Escande, Phys. Plasmas 13, 056102 (2006)

[113] D.F. Escande and M. Ottaviani, Phys. Lett. A 323, 278 (2004).

[114] L. Chacón et al., J. Comput. Phys. 178, 15 (2002); J. Comput. Phys. 188, 573 (2003)

[115] D. Bonfiglio, L. Chacon, S. Cappello, Phys. Plasmas 17, 082501 (2010).

[116] Mirza A. A., Scheffel J., Johnson T., Nucl. Fusion 52,123012 (2012)

[117] Antoni V 2002 Recent Res. Devel. Plasmas 219

[118] M. E. Puiatti et al., Phys. Plasmas 16, 012505 (2009); Nucl. Fusion 49, 045012 (2009). 